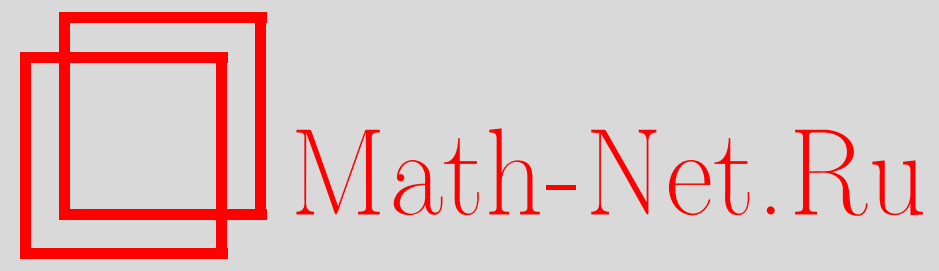

Л. В. Розовский, Большие уклонения типа Чернова для сумм со случайной нормировкой, Теория вероятн. и ее примен., 2008, том 53, выпуск 4, 810-817

DOI: https://doi.org/10.4213/tvp2468

Использование Общероссийского математического портала Math$\mathrm{Net.Ru}$ подразумевает, что вы прочитали и согласны с пользовательским соглашением

http://www . mathnet.ru/rus/agreement

Параметры загрузки:

IP: 52.90 .164 .192

26 апреля 2023 г., 09:49:43

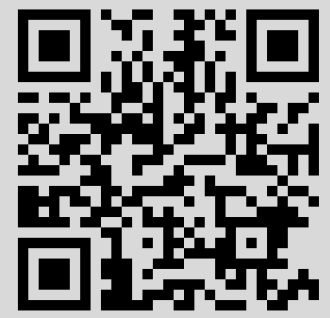


(c) $2008 \mathrm{r}$.

РОЗОВСКИй Л. В.

\section{БОЛЬШИЕ УКЛОНЕНИЯ ТИПА ЧЕРНОВА ДЛЯ СУММ СО СЛУЧАЙНОЙ НОРМИРОВКОЙ}

В заметке изучаются вероятности больших уклонений типа Чернова для сумм независимых одинаково распределенных случайных величин со случайной нормировкой, в том числе самонормированных сумм.

Ключевые слова и фразы: независимые случайные величины, теорема Чернова, самонормированные суммы, большие уклонения.

1. Введение. Пусть случайные величины $\left\{X_{i}\right\}_{i \geqslant 1}$ являются независимыми копиями случайной величины $X$. Положим $S_{n}=X_{1}+\cdots+X_{n}, n \geqslant 1$.

Приведем классический результат, принадлежаший $Г$. Чернову [1] (см. также [2, теорема 3.1]).

Теорема 1. Выполнено соотношение

$$
\lim _{n \rightarrow \infty}\left[\mathbf{P}\left\{S_{n} \geqslant 0\right\}\right]^{1 / n}=\inf _{t \geqslant 0} \mathbf{E} e^{t X} .
$$

Изучая большие уклонения самонормированных сумм независимых одинаково распределенных случайных величин, К.-М. Шао [3] доказал следуюшую теорему.

Теорема 2. Пусть $p>1$ u $V_{n p}=\left(n^{-1} \sum_{i=1}^{n}\left|X_{i}\right|^{p}\right)^{1 / p}$. Предположим, ито

$$
\mathbf{E} X \geqslant 0 \text { uлu } \mathbf{E}|X|^{p}<\infty \text {. }
$$

Тогда

$$
\lim _{n \rightarrow \infty}\left[\mathbf{P}\left\{\frac{S_{n}}{V_{n p}} \geqslant x n\right\}\right]^{1 / n}=\sup _{c \geqslant 0} \inf _{t \geqslant 0} \mathbf{E} \exp \left(t\left(c X-\frac{x}{p}|X|^{p}-x\left(1-\frac{1}{p}\right) c^{p /(p-1)}\right)\right)
$$

npu $x>\mathbf{E} X /\left(\mathbf{E}|X|^{p}\right)^{1 / p}$, где $\mathbf{E} X /\left(\mathbf{E}|X|^{p}\right)^{1 / p}=0$, еслu $\mathbf{E}|X|^{p}=\infty$.

В заметке доказаны утверждения, которые уточняют и обобшают теорему 2. Нами рассматривается последовательность независимых одинаково распределенных случайных пар $\left(X_{i}, Y_{i}\right), i \geqslant 1$, в которьх неотрицательная координата $Y_{i}$ в некотором смысле мажорирует $X_{i}$. Изучены два случая (теоремы 5 и 6 соответственно). В первом из них $S_{n}$ нормируется величиной $n M_{n}$, где $M_{n}=\max _{1 \leqslant i \leqslant n} Y_{i}$. При $Y_{i}=\left|X_{i}\right|$, как следствие теоремы 5 , получаем обобщение результата Шао на случай $p=\infty$.

Теорема 3. При $x \geqslant 0\left(u V_{n \infty}=\max _{1 \leqslant i \leqslant n}\left|X_{i}\right|\right)$

$$
\lim _{n \rightarrow \infty}\left[\mathbf{P}\left\{S_{n} \geqslant x n V_{n \infty}\right\}\right]^{1 / n}=\sup _{v \geqslant 0} \inf _{t \geqslant 0} e^{-t x v} \mathbf{E} e^{t X} 1[|X| \leqslant v]
$$

Отметим, что при $x=0$ соотношения (4) и (1) идентичны. Действительно,

$$
\begin{aligned}
\sup _{v \geqslant 0} \inf _{t \geqslant 0} \mathbf{E} e^{t X} 1[|X| \leqslant v] & =\lim _{n \rightarrow \infty} \inf _{t \geqslant 0} \mathbf{E} e^{t X} 1[|X| \leqslant n] \\
& =\inf _{t \geqslant 0} \lim _{n \rightarrow \infty} \mathbf{E} e^{t X} 1[|X| \leqslant n]=\inf _{t \geqslant 0} \mathbf{E} e^{t X}
\end{aligned}
$$

(см. [1, лемма 4], а также $[4$, гл. 4, § 2]).

Во втором случае нормировка осуществляется величиной $n f\left(\left(Y_{1}+\cdots+Y_{n}\right) / n\right)$, где $f(y), y>0$, принадлежит некоторому классу положительных вогнутьх функщий, содержащему, в частности, функции $y^{r}, 0<r<1$. Формула, описывающая «предельный вид》 больших уклонений, зависит от $f(y)$. Соответствующее следствие теорем 6 и 7 при $Y_{i}=\left|X_{i}\right|^{p}, f(y)=y^{1 / p}(p>1)$ уточняет теорему 2 (сняты условие (2) и ограничения на $x$ ).

1) Работа выполнена при поддержке РФФИ (грант № 06-01-00179-а) и программы «Ведушие научные школы» (грант НШ-4222.2006.1). 
Теорема 4. Пусть $p>1$. В обозначениях теоремы 2 при $x \geqslant 0$

$$
\begin{aligned}
& \lim _{n \rightarrow \infty} {\left[\mathbf{P}\left\{S_{n} \geqslant x n V_{n p}\right\}\right]^{1 / n} } \\
& \quad=\max \left(\sup _{b>0} \inf _{t \geqslant 0} \mathbf{E} \exp \left(t\left(X-\frac{x}{p} b^{1-p}|X|^{p}-x\left(1-\frac{1}{p}\right) b\right)\right), \mathbf{P}\{X=0\}\right) .
\end{aligned}
$$

Заметим, что соотношение (5) (при $p>1$ ) можно переписать в форме (3), а также что равенство (5) остается справедливым и при $p=1$. Последнее, впрочем, не слишком интересно, поскольку имеет место следуюший результат, который вытекает непосредственно из теоремы 1: $n p u$ всех $x$

$$
\lim _{n \rightarrow \infty}\left[\mathbf{P}\left\{S_{n} \geqslant x n V_{n}\right\}\right]^{1 / n}=\inf _{t \geqslant 0} \mathbf{E} \exp (t(X-x Y)) .
$$

В заключение отметим, что при проверке равенства (3) для вероятности $\mathbf{P}\left\{S_{n} \geqslant\right.$ $\left.x n V_{n p}\right\}$ в [3] строятся подходящие оценки снизу и сверху с последуюшим предельным переходом. Рассуждения, примененные при получении соотношений (4), (5) и их обобщений в настоящей работе, проводятся по аналогичной схеме, однако методика доказательств, особенно в части оценки сверху в теореме 5 , использует другие идеи.

2. Результаты. Пусть $(X, Y), Y \geqslant 0,-$ некоторый случайный вектор, а $\left(X_{1}, Y_{1}\right),\left(X_{2}, Y_{2}\right), \ldots$ являются его независимыми копиями. Положим $S_{n}=X_{1}+\cdots+$ $X_{n}, V_{n}=\left(Y_{1}+\cdots+Y_{n}\right) / n, n \geqslant 1$.

Теорема 5. Положим $M_{n}=\max _{1 \leqslant i \leqslant n} Y_{i}, n \geqslant 1$. Если

$$
X \leqslant A Y+a \quad(\exists a, A>0),
$$

mo $n p u x \geqslant 0$

$$
\lim _{n \rightarrow \infty}\left[\mathbf{P}\left\{S_{n} \geqslant x n M_{n}\right\}\right]^{1 / n}=\sup _{v \geqslant 0} \inf _{t \geqslant 0} e^{-t x v} \mathbf{E} e^{t X} 1[Y \leqslant v] .
$$

Теорема 3 является следствием теоремы 5 при $Y=|X|$. Можно также положить $Y=\left|X+x_{0}\right|$ или, скажем, $Y=\max (X, 0)$.

Далее мы приведем еще одно обобщение теоремы 2. Для этого нам понадобятся дополнительные обозначения.

Пусть $G$ обозначает класс выпуклых на $(0, \infty)$ функций $g(h)$ таких, что $g(\infty)=$ $\lim _{h \rightarrow \infty} g(h)=0$ и $0<g(0)=\lim _{h \rightarrow 0} g(h) \leqslant \infty$.

Каждой функции $g \in G$ поставим в соответствие «сопряженную» функцию

$$
f(y)=\inf _{h>0}(h y+g(h)), \quad y \geqslant 0 .
$$

Преобразование (8) является преобразованием типа Лежандра по отношению к $g(h)$ $[5, \S 26]$. При этом $g(h)=\sup _{y>0}(f(y)-h y), h>0$.

Действительно, в силу (8) выполнено неравенство $f(y) \leqslant h y+g(h), h>0$, откуда следует, что $\tilde{g}(h)=\sup _{y>0}(f(y)-h y) \leqslant g(h)$. С другой стороны,

$$
\begin{aligned}
\tilde{g}(h) & =g(h)+\sup _{y>0}\left(\inf _{t>0}(g(t)-g(h)-(-y)(t-h))\right) \\
& \geqslant g(h)+\inf _{t>0}(g(t)-g(h)-\lambda(t-h)),
\end{aligned}
$$

где $\lambda$ - тангенс угла наклона опорной прямой выпуклой функции $g(h)$ в точке $h$. Отсюда в силу свойств выпуклых функций (см. $[4$, гл. V, (8.5)]) получаем требуемую оценку снизу.

Несложно проверить, что функция $f(y)$ вогнута и не убывает, а функция $\bar{f}(y)=$ $f(y) / y$ не возрастает при $y>0$ и, кроме того,

$$
\begin{array}{ll}
f(0)=\lim _{y \rightarrow 0} f(y)=0, & f(\infty)=\lim _{y \rightarrow \infty} f(y)=g(0), \\
\bar{f}(0)=\lim _{y \rightarrow 0} \bar{f}(y)=\sup \{h: g(h)>0\} \leqslant \infty, & \bar{f}(\infty)=\lim _{y \rightarrow \infty} \bar{f}(y)=0 .
\end{array}
$$


Приведем несколько примеров функций $g(h)$ и соответствуюших сопряженных к ним функций $f(y)$ (далее используется обозначение $x^{+}=\max (x, 0)$ ):

1) $g(h)=(1-r)(r / h)^{r /(1-r)}, r \in(0,1)$ и и $f(y)=y^{r}(g(0)=\infty, \bar{f}(0)=\infty)$;

2) $g(h)=(h-\ln h-1) 1[h \leqslant 1]$ и $f(y)=\ln (1+y)(g(0)=\infty, \bar{f}(0)=1)$;

3) $g(h)=e^{1 / c}(c-h)^{+}, c=\left(1-\sqrt{(1-4 h)^{+}}\right) / 2$, и $f(y)=y / \max (2, \ln y)(g(0)=\infty$, $\bar{f}(0)=1 / 2)$;

4) $g(h)=\left(1-h^{r}\right)^{+}, r \in(0,1]$, и $f(y)=y, 0<y \leqslant r ; f(y)=1-(1-r)(r / y)^{r /(1-r)}$, $y>r(g(0)=1, \bar{f}(0)=1)$;

5) $g(h)=e^{-h}$ и $f(y)=y \ln (e / y), 0<y \leqslant 1 ; f(y)=1, y>1(g(0)=1, \bar{f}(0)=\infty)$.

Пусть $g \in G$, а $f$ определена формулой (8).

Теорема 6. Предположим, ито при любых положительных $t$ и $\varepsilon$

$$
\mathbf{E} e^{t(X-\varepsilon Y)}<\infty
$$

и, кроме того,

$$
g(0)<\infty \quad \text { или } \mathbf{E} e^{t X}<\infty \quad(\exists t>0)
$$

Toгда прu $x \geqslant 0$

$$
\begin{aligned}
\lim _{n \rightarrow \infty} & {\left[\mathbf{P}\left\{S_{n} \geqslant x n f\left(V_{n}\right)\right\}\right]^{1 / n} } \\
& =\max \left(\inf _{t \geqslant 0} \mathbf{E} e^{t X} 1[Y=0], \sup _{h \geqslant 0} \inf _{t \geqslant 0} \mathbf{E} e^{t(X-x h Y-x g(h))}\right) .
\end{aligned}
$$

Здесь предполагаетсs, ито $\inf _{t \geqslant 0} \mathbf{E} e^{t(X-x g(0))}=0$, ecли $g(0)=\infty$.

Условие (10) удовлетворяется, когда $\mathbf{E} e^{t X}<\infty(\forall t>0)$ или когда $X \leqslant a Y+c$ $\left(\forall a>0 \exists c>0\right.$ ) (как в случае $Y=\left|X+x_{0}\right|^{p}$ или $Y=\left(X^{+}\right)^{p}$, где постоянная $p$ строго больше 1).

Рассмотрим теперь частный случай ситуации, при которой условие (11) не выполняется (более обший, имеюший предварительный характер, результат содержит лемма из п. 4).

Пусть $p>1$, а функция $\tau(y)$ удовлетворяет условиям

$$
\limsup _{y \rightarrow \infty} \frac{\tau(y)}{y}<\infty \quad \text { и } \quad \liminf _{y \rightarrow \infty} \tau(y)>0 .
$$

Теорема 7. Предположим, что

$$
\liminf _{h \rightarrow 0} g(h) h^{1 /(p-1)}>0
$$

(или, равносильно, $\liminf _{y \rightarrow \infty} f(y) / y^{1 / p}>0$ ) и при некотором положительном $k$

$$
\begin{aligned}
& \mathbf{E} e^{t \tau(X)} \mathbf{1}[X>k]<\infty \quad(\forall t>0), \\
& Y \geqslant A \frac{X^{p}}{\tau^{p-1}(X)} \mathbb{1}[X>k] \quad(\exists A>0) .
\end{aligned}
$$

Тогда при $x \geqslant 0$ справедливо соотношение (12).

Заметим, что если в (13)

$$
\liminf _{h \rightarrow 0} g(h) h^{1 /(p-1)}=\infty, \quad \text { или } \quad \liminf _{y \rightarrow \infty} \frac{f(y)}{y^{1 / p}}=\infty,
$$

то в условии (14) достаточно предполагать, что $\mathbf{E} e^{t \tau(X)} \mathbf{1}[X>k]<\infty(\exists t>0)$.

Отметим также, что в случае $\lim \sup _{y \rightarrow \infty} \tau(y)<\infty$ условие (14) всегда выполняется.

Теорема 4 вытекает из теорем 6 и 7 при $Y=|X|^{p}, f(y)=y^{1 / p}$ и $\tau(y)=1, y>0$. 


\section{3. Доказательство теоремы 5.}

О ц ен к а с н и з у. Пусть $v \geqslant 0$ и $x>0$. Тогда

$$
\begin{aligned}
P_{n}(x) & =\mathbf{P}\left\{S_{n} \geqslant x n M_{n}\right\} \geqslant \mathbf{P}\left\{S_{n} \geqslant x n M_{n}, M_{n} \leqslant v\right\} \\
& \geqslant P_{n}(x, v)=\mathbf{P}\left\{S_{n} \geqslant x n v, M_{n} \leqslant v\right\} .
\end{aligned}
$$

Следовательно,

$$
\liminf _{n \rightarrow \infty} P_{n}(x)^{1 / n} \geqslant \liminf _{n \rightarrow \infty} \sup _{v \geqslant 0} P_{n}(x, v)^{1 / n} \geqslant \sup _{v \geqslant 0} \liminf _{n \rightarrow \infty} P_{n}(x, v)^{1 / n} .
$$

По теореме 1

$$
\liminf _{n \rightarrow \infty} P_{n}(x, v)^{1 / n}=\lim _{n \rightarrow \infty} P_{n}(x, v)^{1 / n}=\inf _{t \geqslant 0} M_{v}(t, x),
$$

где

$$
M_{v}(t, x)=e^{-t x v} \mathbf{E} e^{t X} 1[Y \leqslant v]
$$

откуда

$$
\liminf _{n \rightarrow \infty}\left[\mathbf{P}\left\{S_{n} \geqslant x n M_{n}\right\}\right]^{1 / n} \geqslant \sup _{v \geqslant 0} \inf _{t \geqslant 0} e^{-t x v} \mathbf{E} e^{t X} \mathbf{1}[Y \leqslant v] .
$$

О ц е н к а с в е р х у. Согласно (1),

$$
\begin{aligned}
P_{n}(x) & =\mathbf{P}\left\{S_{n} \geqslant x n M_{n}, \bigcup_{i=1}^{n}\left\{M_{n}=Y_{i}\right\}\right\} \\
& \leqslant n \mathbf{P}\left\{S_{n-1} \geqslant x n M_{n}-X_{n}, M_{n-1} \leqslant Y_{n}\right\} \\
& \leqslant n \mathbf{P}\left\{S_{n-1} \geqslant x n Y_{n}-A Y_{n}-a, M_{n-1} \leqslant Y_{n}\right\} \\
& \leqslant n \sup _{v \geqslant 0} \mathbf{P}\left\{S_{n-1} \geqslant(x n-A) v-a, M_{n-1} \leqslant v\right\} \\
& \leqslant n \sup _{v \geqslant 0}\left(\inf _{t \geqslant 0} \exp \left(-t \frac{(x n-A) v-a}{n-1}\right) \mathbf{E} e^{t X} \mathbf{1}[Y \leqslant v]\right)^{n-1} .
\end{aligned}
$$

Пусть $\varepsilon>0$. Из (20) следует, что

$$
\limsup _{n \rightarrow \infty} P_{n}(x)^{1 /(n-1)} \leqslant \max _{i=1,2,3} \limsup _{n \rightarrow \infty} I_{n, i}(\varepsilon),
$$

где

$$
\begin{aligned}
& I_{n, 1}(\varepsilon)=\sup _{0 \leqslant v<\varepsilon} \inf _{t \geqslant 0} \exp \left(t \frac{a}{n-1}\right) \mathbf{E} e^{t X} \mathbf{1}[Y \leqslant v], \\
& I_{n, 2}(\varepsilon)=\sup _{\varepsilon \leqslant v \leqslant 1 / \varepsilon} \inf _{t \geqslant 0} \exp \left(-t v \frac{x n-A-a / \varepsilon}{n-1}\right) \mathbf{E} e^{t X} \mathbf{1}[Y \leqslant v], \\
& I_{n, 3}(\varepsilon)=\sup _{v>1 / \varepsilon} \inf _{t \geqslant 0} \exp \left(-t v \frac{x n-A-a \varepsilon}{n-1}\right) \mathbf{E} e^{t X} 1[Y \leqslant v] .
\end{aligned}
$$

Положим $f_{\varepsilon}(\delta)=\inf _{t \geqslant 0} e^{-t \delta} \mathbf{E} e^{t X} \mathbf{1}[Y \leqslant \varepsilon]$. Эта функция при всех $\delta$ является невозрастаюшей и непрерывной слева (см. $[1$, доказательство леммы 6]). Отсюда при $\delta_{n}=-a /(n-1)$ получаем

$$
\begin{aligned}
\limsup _{n \rightarrow \infty} I_{n, 1}(\varepsilon) & \leqslant \limsup _{n \rightarrow \infty} f_{\varepsilon}\left(\delta_{n}\right)=\lim _{n \rightarrow \infty} f_{\varepsilon}\left(\delta_{n}\right) \\
& =f_{\varepsilon}(-0)=f_{\varepsilon}(0)=\inf _{t \geqslant 0} \mathbf{E} e^{t X} \mathbf{1}[Y \leqslant \varepsilon] .
\end{aligned}
$$

Далее, в силу (6) при любом $c \geqslant a$ и $y>0$

$$
\begin{aligned}
M_{v}(t, y) & =e^{-t v y} \mathbf{E} e^{t X}(\mathbf{1}[X \leqslant c, Y \leqslant v]+1[X>c, Y \leqslant v]) \\
& \leqslant e^{-t v y}\left(e^{t c}+\mathbf{E} e^{t A Y} 1[Y \leqslant v]\right) \leqslant e^{-t v y}\left(e^{t c}+e^{t A v} R(c)\right),
\end{aligned}
$$


где $R(c)=\mathbf{P}\{X \geqslant c\}$, а $M_{v}(t, y)$ определено в (18). При $c=y v / 2 \geqslant a$ отсюда следует неравенство

$$
\inf _{t \geqslant 0} M_{v}(t, y) \leqslant 2 R(v y / 2)^{y /(2 A)}
$$

и, таким образом, при любом $\varepsilon<x /(2 A)$

$$
\limsup _{n \rightarrow \infty} I_{n, 3}(\varepsilon) \leqslant 2 R\left(\frac{x}{2 \varepsilon}\right)^{x /(2 A)} .
$$

Теперь займемся $I_{n, 2}(\varepsilon)$. Обозначим $\delta_{n}=(A-x+a / \varepsilon) /(n-1), \bar{M}_{v}(y)=$ $\inf _{t \geqslant 0} M_{v}(t, y)$. Без потери обшности полагая $\delta_{n}>0$ и имея в виду, что последовательность $m_{n}(\varepsilon)=\sup _{\varepsilon \leqslant v \leqslant 1 / \varepsilon} \bar{M}_{v}\left(x-\delta_{n}\right)$ не возрастает, находим

$$
\limsup _{n \rightarrow \infty} I_{n, 2}(\varepsilon)=\limsup _{n \rightarrow \infty} m_{n}(\varepsilon)=\lim _{n \rightarrow \infty} m_{n}(\varepsilon)=\liminf _{n \rightarrow \infty} m_{n}(\varepsilon) .
$$

Поскольку $m_{n}(\varepsilon) \leqslant \varepsilon+\bar{M}_{v_{n}}\left(x-\delta_{n}\right)\left(\exists v_{n}: \varepsilon \leqslant v_{n} \leqslant 1 / \varepsilon\right)$, то

$$
\liminf _{n \rightarrow \infty} m_{n}(\varepsilon) \leqslant \varepsilon+\liminf _{n \rightarrow \infty} \bar{M}_{v_{n}}\left(x-\delta_{n}\right) \text {. }
$$

Выберем $v^{\prime}=v_{n_{k}} \rightarrow v_{0} \in[\varepsilon, 1 / \varepsilon]$. Без потери общности можно считать, что подпоследовательность $v^{\prime}$ монотонна. Положим $\delta^{\prime}=\delta_{n_{k}}$.

Если $v^{\prime} \nearrow v_{0}$, то

$$
\bar{M}_{v^{\prime}}\left(x-\delta^{\prime}\right) \leqslant \inf _{t \geqslant 0} e^{-t v^{\prime}\left(x-\delta^{\prime}\right)} \mathbf{E} e^{t X} \mathbf{1}\left[Y \leqslant v_{0}\right]=\bar{M}_{v_{0}}\left(t, x-\delta^{\prime \prime}\right),
$$

где $\delta^{\prime \prime}=\delta^{\prime}+\left(x-\delta^{\prime}\right)\left(v_{0}-v^{\prime}\right) / v_{0} \searrow 0$. Поэтому, так же как в (22),

$$
\liminf _{n \rightarrow \infty} \bar{M}_{v_{n}}\left(x-\delta_{n}\right) \leqslant \liminf _{k \rightarrow \infty} \bar{M}_{v^{\prime}}\left(x-\delta^{\prime}\right) \leqslant \liminf _{k \rightarrow \infty} \bar{M}_{v_{0}}\left(x-\delta^{\prime \prime}\right)=\bar{M}_{v_{0}}(x) .
$$

Таким образом,

$$
\limsup _{n \rightarrow \infty} I_{n, 2}(\varepsilon) \leqslant \varepsilon+\bar{M}_{v_{0}}(x) \leqslant \varepsilon+\sup _{\varepsilon \leqslant v \leqslant 1 / \varepsilon} \bar{M}_{v}(x) .
$$

Пусть теперь $v^{\prime} \searrow v_{0}$. Так же как в $[1,(4.16)]$, найдется конечное $t^{\prime}$ такое, что $\bar{M}_{v_{0}}(x) \geqslant M_{v_{0}}\left(t^{\prime}, x\right)-\varepsilon$. Тогда в силу (6)

$$
\begin{aligned}
\bar{M}_{v^{\prime}}\left(x-\delta^{\prime}\right) & \leqslant e^{-t^{\prime} v_{0}\left(x-\delta^{\prime}\right)}\left(\mathbf{E} e^{t^{\prime} X} \mathbf{1}\left[Y \leqslant v_{0}\right]+e^{t^{\prime}\left(a+A v^{\prime}\right)} \mathbf{E} \mathbf{1}\left[v_{0}<Y \leqslant v^{\prime}\right]\right) \\
& =e^{t^{\prime} v_{0} \delta^{\prime}} M_{v_{0}}\left(t^{\prime}, x\right)+e^{t^{\prime}\left(a+A v^{\prime}-v_{0}\left(x-\delta^{\prime}\right)\right)} \mathbf{P}\left\{v_{0}<Y \leqslant v^{\prime}\right\} .
\end{aligned}
$$

Отсюда вытекают соотношение

$$
\liminf _{n \rightarrow \infty} \bar{M}_{v_{n}}\left(x-\delta_{n}\right) \leqslant M_{v_{0}}\left(t^{\prime}, x\right) \leqslant \varepsilon+\bar{M}_{v_{0}}(x)
$$

и, аналогично предыдушему случаю, оценка (24).

Из (21)-(23) и (24) получим при всех достаточно малых положительных $\varepsilon$

$$
\limsup _{n \rightarrow \infty} P_{n}(x)^{1 /(n-1)} \leqslant \max \left(f_{\varepsilon}(0), \varepsilon+\sup _{0<v<\infty} \bar{M}_{v}(x), 2 R\left(\frac{x}{2 \varepsilon}\right)^{x /(2 A)}\right) .
$$

Выберем теперь конечное $t^{\prime}$ так, чтобы $f_{0}(0) \geqslant \mathbf{E} e^{t^{\prime} X} \mathbf{1}[Y=0]-\varepsilon$. Тогда

$$
\begin{aligned}
f_{\varepsilon}(0) & \leqslant \mathbf{E} e^{t^{\prime} X} 1[Y=0]+\mathbf{E} e^{t^{\prime} X} 1[0<Y \leqslant \varepsilon] \\
& \leqslant \varepsilon+f_{\varepsilon}(0)+e^{t^{\prime}(a+\varepsilon A)} \mathbf{P}\{0<Y \leqslant \varepsilon\} .
\end{aligned}
$$

В силу произвольной малости $\varepsilon,(25)$ и (26) приводят к соотношению

откуда

$$
\limsup _{n \rightarrow \infty} P_{n}(x)^{1 /(n-1)} \leqslant \max \left(f_{0}(0), \sup _{0<v<\infty} \bar{M}_{v}(x)\right)=\sup _{0 \leqslant v<\infty} \bar{M}_{v}(x),
$$

$$
\limsup _{n \rightarrow \infty} P_{n}(x)^{1 / n} \leqslant \sup _{0 \leqslant v<\infty} \bar{M}_{v}(x) .
$$

Теорема 5 при $x>0$ следует из (19) и (27), а при $x=0$ из (1). 
4. Доказательство теорем 6 и 7. Пусть $g \in G$, а $f$ определена формулой (8), $x>0$. Положим

$$
\begin{aligned}
P_{n}(x) & =\mathbf{P}\left\{S_{n} \geqslant x n f\left(V_{n}\right)\right\} ; \\
P_{(0, \infty)} & =\sup _{h>0} \inf _{t \geqslant 0} \mathbf{E} e^{t(X-x h Y-x g(h))}, \quad P_{\infty}=\inf _{t \geqslant 0} \mathbf{E} e^{t X} \mathbf{1}[Y=0] ; \\
P_{0} & =\inf _{t \geqslant 0} \mathbf{E} e^{t(X-x g(0))}, \text { если } g(0)<\infty, \quad \text { и } P_{0}=0, \text { если } g(0)=\infty ; \\
\bar{P} & =\lim _{\varepsilon \rightarrow 0} \limsup _{n \rightarrow \infty}\left[\mathbf{P}\left\{S_{n} \geqslant x n \inf _{0<h<\varepsilon}\left(h V_{n}+g(h)\right)\right\}\right]^{1 / n} ; \\
\underline{P} & =\lim _{\varepsilon \rightarrow 0} \liminf _{n \rightarrow \infty}\left[\mathbf{P}\left\{S_{n} \geqslant x n \inf _{0<h<\varepsilon}\left(h V_{n}+g(h)\right)\right\}\right]^{1 / n} .
\end{aligned}
$$

Лемма. Имеют место неравенства

$$
\begin{aligned}
& \liminf _{n \rightarrow \infty} P_{n}(x)^{1 / n} \geqslant \max \left(P_{0}, P_{(0, \infty)}, P_{\infty}, \underline{P}\right), \\
& \limsup _{n \rightarrow \infty} P_{n}(x)^{1 / n} \leqslant \max \left(P_{0}, P_{(0, \infty)}, P_{\infty}, \bar{P}\right) .
\end{aligned}
$$

Д ок а з ате ль ст в о ле м мы. Оченка снизу. Имеем:

$$
\begin{aligned}
P_{n}(x) \geqslant & \mathbf{P}\left\{S_{n} \geqslant x n f(\infty)\right\}=\mathbf{P}\left\{\sum_{i=1}^{n}\left(X_{i}-x g(0)\right) \geqslant 0\right\} \\
& (f(\infty)=g(0)<\infty) ; \\
P_{n}(x) \geqslant & \mathbf{P}\left\{S_{n} \geqslant x n f(0), V_{n}=0\right\}=\mathbf{P}\left\{S_{n} \geqslant 0, \bigcap_{i=1}^{n}\left\{Y_{i}=0\right\}\right\} ; \\
P_{n}(x)= & \mathbf{P}\left\{S_{n} \geqslant x n \inf _{h>0}\left(h V_{n}+g(h)\right)\right\} \\
\geqslant & \sup _{h>0} \mathbf{P}\left\{\sum_{i=1}^{n}\left(X_{i}-x h Y_{i}-x g(h)\right) \geqslant 0\right\} ; \\
P_{n}(x) \geqslant & \mathbf{P}\left\{S_{n} \geqslant x n \inf _{0<h<\varepsilon}\left(h V_{n}+g(h)\right)\right\}, \quad \varepsilon>0 .
\end{aligned}
$$

Оценка (28) следует из (30) и теоремы 1.

Оченка сверху. Пусть $0<\varepsilon<1$ и $\delta=\bar{f}(\varepsilon)-\varepsilon>0$. Тогда

$$
P_{n}(x) \leqslant I_{1}+I_{2}+I_{3}
$$

где

$$
\begin{aligned}
& I_{1}=\mathbf{P}\left\{S_{n} \geqslant x n \inf _{0<h<\varepsilon}\left(h V_{n}+g(h)\right)\right\}, \\
& I_{2}=\mathbf{P}\left\{S_{n} \geqslant x n \inf _{h>\bar{f}(\varepsilon)}\left(h V_{n}+g(h)\right)\right\}, \\
& I_{3}=\mathbf{P}\left\{S_{n} \geqslant x n \inf _{\varepsilon \leqslant h \leqslant \bar{f}(\varepsilon)}\left(h V_{n}+g(h)\right)\right\} .
\end{aligned}
$$

Имеем:

$$
\begin{aligned}
& I_{2} \leqslant \mathbf{P}\left\{S_{n} \geqslant x n \bar{f}(\varepsilon) Y\right\} \leqslant\left(\inf _{t \geqslant 0} \mathbf{E} e^{t(X-x \bar{f}(\varepsilon) Y)}\right)^{n}=\mu^{n}(\varepsilon) \\
& I_{3}=\mathbf{P}\left\{\sup _{\varepsilon \leqslant h \leqslant \bar{f}(\varepsilon)} \sum_{i=1}^{n}\left(X_{i}-x h Y_{i}-x g(h)\right) \geqslant 0\right\} .
\end{aligned}
$$


Оценим $I_{3}$. Для $k=0,1, \ldots, n$ положим $h_{k}=\varepsilon+k \delta / n, \Delta_{n}=\max _{0 \leqslant k<n}\left(g\left(h_{k}\right)-\right.$ $\left.g\left(h_{k+1}\right)\right)$ (заметим, что $\lim _{n \rightarrow \infty} \Delta_{n}=0$ ). В силу (34) имеем:

$$
\begin{aligned}
I_{3} & \leqslant \sum_{0 \leqslant k<n} \mathbf{P}\left\{\sup _{h_{k} \leqslant h \leqslant h_{k+1}} \sum_{i=1}^{n}\left(X_{i}-x h Y_{i}-x g(h)\right) \geqslant 0\right\} \\
& \leqslant n \max _{0 \leqslant k<n} \mathbf{P}\left\{\sum_{i=1}^{n}\left(X_{i}-x h_{k} Y_{i}-x g\left(h_{k+1}\right)\right) \geqslant 0\right\} \\
& \leqslant n \max _{1 \leqslant k \leqslant n} \mathbf{P}\left\{\sum_{i=1}^{n}\left(X_{i}-x h_{k} Y_{i}-x g\left(h_{k}\right)+x \Delta_{n}\right) \geqslant 0\right\} \\
& \leqslant n \sup _{\varepsilon \leqslant h \leqslant \bar{f}(\varepsilon)} \mathbf{P}\left\{\sum_{i=1}^{n}\left(X_{i}-x h Y_{i}-x g(h)+x \Delta_{n}\right) \geqslant 0\right\} \\
& \leqslant n\left(\sup _{\epsilon \leqslant h \leqslant \bar{f}(\varepsilon)} \inf _{t \geqslant 0} \mathbf{E} e^{t\left(X-x h Y-x g(h)+x \Delta_{n}\right)}\right)^{n}=n \beta_{n}^{n} .
\end{aligned}
$$

Из (31)-(35) следует

$$
\begin{aligned}
\limsup _{n \rightarrow \infty} P_{n}(x)^{1 / n} & \leqslant \limsup _{n \rightarrow \infty}\left(\mu^{n}(\varepsilon)+I_{1}+n \beta_{n}^{n}\right)^{1 / n} \\
& \leqslant \limsup _{n \rightarrow \infty} 3^{1 / n} \max \left(\mu(\varepsilon), I_{1}^{1 / n}, n^{1 / n} \beta_{n}\right) \\
& =\max \left(\mu(\varepsilon), \bar{P}(\varepsilon), \tau(\varepsilon), \lim _{n \rightarrow \infty} \beta_{n}\right)
\end{aligned}
$$

где $\bar{P}(\varepsilon)=\lim \sup _{n \rightarrow \infty}\left[\mathbf{P}\left\{S_{n} \geqslant x n \inf _{0<h<\varepsilon}\left(h V_{n}+g(h)\right)\right\}\right]^{1 / n}$.

Обозначим $Z(h)=X-x h Y-x g(h), h>0$, и пусть $M(h)=\inf _{t \geqslant 0} \mathbf{E} e^{t Z(h)}$.

Предел $\lim _{n \rightarrow \infty} \beta_{n}$ сушествует (и больше или равен $\sup _{\varepsilon \leqslant h \leqslant \bar{f}(\varepsilon)} M(h)$ ). Следовательно, найдется растушая к бесконечности подпоследовательность $n^{\prime}$ такая, что при фиксированном $\varepsilon^{\prime}>0$ выполнено $h_{n^{\prime}} \rightarrow h_{0} \in[\varepsilon, \bar{f}(\varepsilon)]$ и

$$
\beta_{n^{\prime}} \leqslant \varepsilon^{\prime}+M_{n^{\prime}}\left(h_{n^{\prime}}\right) \text {, }
$$

где $M_{n^{\prime}}\left(h_{n^{\prime}}\right)=\inf _{t \geqslant 0} \mathbf{E} e^{t\left(Z\left(h_{n^{\prime}}\right)+x \Delta_{n^{\prime}}\right)}$.

В силу условия (9) и принципа мажорируемой сходимости (см. $[4$, гл. $4, \S 2]$ )

$$
\begin{aligned}
\lim _{n^{\prime}} M_{n^{\prime}}\left(h_{n^{\prime}}\right) & \leqslant \inf _{t \geqslant 0} \lim _{n^{\prime}} \mathbf{E} e^{t\left(Z\left(h_{n^{\prime}}\right)+x \Delta_{n^{\prime}}\right)} \\
& =\inf _{t \geqslant 0} \mathbf{E} \lim _{n^{\prime}} e^{t\left(Z\left(h_{n^{\prime}}\right)+x \Delta_{n^{\prime}}\right)}=M\left(h_{0}\right) \leqslant \sup _{\varepsilon \leqslant h \leqslant \bar{f}(\varepsilon)} M(h) .
\end{aligned}
$$

Из (38) и (37) в силу произвольности $\varepsilon^{\prime}$ следует

$$
\lim _{n \rightarrow \infty} \beta_{n}=\sup _{\varepsilon \leqslant h \leqslant \bar{f}(\varepsilon)} M(h) \leqslant \sup _{0<h<\infty} M(h) .
$$

Теперь, устремляя в (36) $\varepsilon$ к нулю, аналогично (38) получим

$$
\lim _{\varepsilon \rightarrow 0} \mu(\varepsilon) \leqslant \inf _{t \geqslant 0} \lim _{\varepsilon \rightarrow 0} \mathbf{E} e^{t(X-x \bar{f}(\varepsilon) Y)}=\inf _{t \geqslant 0} \mathbf{E} \lim _{\varepsilon \rightarrow 0} e^{t(X-x \bar{f}(\varepsilon) Y)}=\widetilde{P},
$$

где $\widetilde{P}=\inf _{t \geqslant 0} \mathbf{E} e^{t(X-x \bar{f}(0) Y)} \leqslant \sup _{0<h<\infty} M(h)$, когда $\bar{f}(0)<\infty\left(\right.$ см. (9)), и $\widetilde{P}=P_{\infty}$ в противном случае.

Оценка (29) следует из (36), (39), (40). Лемма доказана.

Проверим теорему 6 . При $x=0$ соотношение (12) вытекает из теоремы 1.

Пусть $x>0$. Если $g(0)<\infty$, то

$$
\underline{P} \geqslant \liminf _{n \rightarrow \infty}\left[\mathbf{P}\left\{S_{n} \geqslant x n g(0)\right\}\right]^{1 / n}=\inf _{t \geqslant 0} \mathbf{E} e^{t(X-x g(0))}
$$


и

$$
\begin{aligned}
\bar{P} & \leqslant \lim _{\varepsilon \rightarrow 0} \limsup _{n \rightarrow \infty}\left[\mathbf{P}\left\{S_{n} \geqslant x n g(\varepsilon)\right\}\right]^{1 / n} \leqslant \lim _{\varepsilon \rightarrow 0} \inf _{t \geqslant 0} \mathbf{E} e^{t(X-x g(\varepsilon))} \\
& =\lim _{\varepsilon \rightarrow 0} \inf _{t \geqslant 0} e^{t x(g(0)-g(\varepsilon))} \mathbf{E} e^{t(X-x g(0))}=\inf _{t \geqslant 0} \mathbf{E} e^{t(X-x g(0))},
\end{aligned}
$$

аналогично (22). Применяя лемму, отсюда получим (12).

Пусть теперь $g(0)=\infty$ и $\mathbf{E} e^{t_{0} X}<\infty, t_{0}>0$. Тогда

$$
\bar{P} \leqslant \lim _{\varepsilon \rightarrow 0} \mathbf{E} e^{t_{0}(X-x g(\varepsilon))}=\lim _{\varepsilon \rightarrow 0} e^{-t_{0} x g(\varepsilon)} \mathbf{E} e^{t_{0} X}=0 .
$$

Используя лемму, отсюда снова получим (12).

Теорема 6 , таким образом, полностью доказана.

Перейдем к доказательству теоремы 7. Мы покажем, что в ее условиях $\bar{P}=0$, а далее воспользуемся леммой. При $k=x g(\varepsilon)$, где положительное $\varepsilon$ достаточно мало, положив $\bar{S}_{n}=\sum_{i=1}^{n} X_{i} \mathbf{1}\left[X_{i}>k\right]$, найдем

$$
\begin{aligned}
\mathbf{P}\left\{S_{n} \geqslant x n \inf _{0<h<\varepsilon}\left(h V_{n}+g(h)\right)\right\} & \leqslant \mathbf{P}\left\{S_{n} \geqslant x n \max \left(g(\varepsilon), f\left(V_{n}\right)\right)\right\} \\
& \leqslant \mathbf{P}\left\{\bar{S}_{n} \geqslant x n f\left(V_{n}\right) / 2\right\} .
\end{aligned}
$$

Далее, аналогично [3, с. 289], по неравенству Гёльдера

$$
\bar{S}_{n} \leqslant\left(\sum_{i=1}^{n} \frac{X_{i}^{p}}{\tau\left(X_{i}\right)^{p-1}} \mathbf{1}\left[X_{i}>k\right]\right)^{1 / p}\left(\sum_{i=1}^{n} \tau\left(X_{i}\right) \mathbf{1}\left[X_{i}>k\right]\right)^{(p-1) / p} .
$$

Из (41), (42) и условий (13), (15) следует, что

$$
\mathbf{P}\left\{S_{n} \geqslant x n \inf _{0<h<\varepsilon}\left(h V_{n}+g(h)\right)\right\} \leqslant \mathbf{P}\left\{\sum_{i=1}^{n} \tau\left(X_{i}\right) \mathbf{1}\left[X_{i}>k\right] \geqslant \delta n\right\}
$$

при некотором положительном $\delta$, не зависящем от $n$ и $\varepsilon$.

Отсюда, воспользовавшись условием (14), заключаем, что

$$
\bar{P} \leqslant \lim _{k \rightarrow \infty} \inf _{t \geqslant 0} \mathbf{E} e^{t(\tau(X) \mathbf{1}[X>k]-\delta)}=0
$$

(выбираем $t$ так, чтобы $e^{-t \delta}$ было мало, и $k$ так, чтобы выполнялось неравенство $\left.\mathbf{E} e^{t \tau(X)} \mathbf{1}[X>k] \leqslant 1\right)$.

Замечание к теореме 7 проверяется аналогично.

\section{СПИСОК ЛИТЕРАТУРЫ}

1. Chernoff $H$. A measure of asymptotic efficiency for tests of a hypothesis based on the sum of observations. - Ann. Math. Statist., 1952, v. 23, № 4, p. 493-507.

2. Bahadur R. R. Some Limit Theorems in Statistics. Philadelphia: SIAM, 1971, 42 p.

3. Shao Q.-M. Self-normalized large deviations. - Ann. Probab., 1997, v. 25, № 1, p. 285-328.

4. Феллер В. Введение в теорию вероятностей и ее приложения. Т. 2. М.: Мир, 1984, $751 \mathrm{c}$.

5. Рокафеллар Р. Выпуклый анализ. М.: Мир, 1973, 469 с.

Поступила в редакцию

27.IX.2006

Исправленный вариант

6.II.2008 